\title{
Volumetric Alterations in the Nucleus Accumbens and Caudate Nucleus in Bulimia Nervosa: A Structural Magnetic Resonance Imaging Study
}

\author{
Joana Coutinho, $\mathrm{PhD}^{1 *}$ \\ Ana Filipa Ramos, MSc ${ }^{1}$ \\ Liliana Maia, $\mathrm{MSc}^{1}$ \\ Liliana Castro, $\mathrm{PhD}^{2,3}$ \\ Eva Conceição, $\mathrm{PhD}^{2}$ \\ Allan Geliebter, $\mathrm{PhD}^{4,5}$ \\ Paulo P.P. Machado, $\mathrm{PhD}^{2}$ \\ Óscar Gonçalves, PhD $^{1,6}$ \\ Adriana Sampaio, $\mathrm{PhD}^{1}$
}

\begin{abstract}
Objective: Bulimia nervosa ( $\mathrm{BN}$ ) is an eating disorder characterized by recurrent episodes of binge eating and inappropriate compensatory behaviors (such as purging, fasting, or excessive exercise) to prevent weight gain. BN has been associated with deficits in inhibitory control processes. The basal ganglia specifically, the nucleus accumbens (NAc) and the caudate nucleus $(\mathrm{CN})$ are part of the frontostriatal circuits involved in inhibitory control. The main goal of this study was to investigate the presence of morphological alterations in the NAC and the CN in a sample of patients diagnosed with $\mathrm{BN}$.

Method: Forty-one female participants, 21 diagnosed with $\mathrm{BN}$ and 20 healthy matched controls $(\mathrm{HC})$, underwent a structural magnetic resonance imaging (MRI) acquisition and clinical assessment.
\end{abstract}

The NAC and the CN were manually segmented using the software Slicer 3D.

Results: The results reveal a significant volumetric decrease in the $\mathrm{CN}$ and a preserved NAC volume in $\mathrm{BN}$ compared to the control group.

Discussion: These findings suggest a contributory role of the caudate nucleus part of the dorsal striatum in the psychopathology of BN. (C) 2014 Wiley Periodicals, Inc.

Keywords: bulimia nervosa; magnetic resonance imaging; manual segmentation; nucleus accumbens; caudate nucleus

(Int J Eat Disord 2015; 48:206-214)

\section{Introduction}

Bulimia nervosa (BN) is an eating disorder characterized by recurrent episodes of binge eating and inappropriate compensatory behaviors (such as purging, fasting, or excessive exercise) to prevent

\footnotetext{
Accepted 25 February 2014

Supported by SFRH/BPD/75014/2010 from Foundation for Science and Technology, Portugal (FCT), by SFRH/SINTD/60106/2009 from Foundation for Science and Technology, Portugal (FCT) and by Foundation for Science and Technology, Portugal (FCT/PTDC/ PSI-PCL/099981/2008).

*Correspondence to: Joana Fernandes Pereira Coutinho, School of Psychology, University of Minho, Campus de Gualtar 4710-057 Braga, Portugal. E-mail: joanafpc@gmail.com

${ }^{1}$ Neuropsychophysiology Lab, CIPsi, School of Psychology, University of Minho, Braga, Portugal

2 Psychotherapy and Psychopathology Research Unit, CIPsi, School of Psychology, University of Minho, Braga, Portugal

${ }^{3}$ Hospital de Magalhães Lemos EPE, Porto, Portugal

${ }^{4}$ NY Obesity Nutrition Research Center, St Luke's-Roosevelt Hospital, Columbia University College of Physicians and Surgeons, New York

${ }^{5}$ Department of Psychology, Touro College and University System, New York

${ }^{6}$ Department of Counseling and Applied Educational Psychology, Bouvé College of Health Sciences, Northeastern University, Boston, Massachusetts

Published online 16 March 2014 in Wiley Online Library (wileyonlinelibrary.com). DOI: 10.1002/eat.22273

(c) 2014 Wiley Periodicals, Inc.
}

weight gain. ${ }^{1}$ Neurocognitive deficits have also been documented in $\mathrm{BN}$, namely impairment in self-regulatory processes, inhibitory control, as well as alterations in the sensitivity to reward/punishment systems that may underlie the impulsive eating behavior. ${ }^{2-5}$ Self-regulatory, inhibitory control, and reward/punishment systems depend upon the integrity of frontostriatal circuits. Accordingly, studies have proposed that the ventral striatum (VST) (i.e., nucleus accumbens-NAc, involved in reward) and the dorsal striatum (caudate nucleus- $\mathrm{CN}$, involved in self-regulation and inhibitory control $)^{6,7}$ may be neurobiological substrates for these symptoms in BN. ${ }^{3}$

More specifically, the NAc is part of the brain's reward system ${ }^{8}$ involved in the processing of pleasurable stimuli such as food. ${ }^{9,10}$ Food reward is processed by the mesolimbic dopaminergic circuit, in which neurons from the brain stem project to the NAc and the orbitofrontal cortex (OBF), ${ }^{11-13}$ regulating reward-directed behavior. ${ }^{14}$ Anatomical alterations in brain regions involved in rewardlearning circuits have been found in other psychiatric disorders characterized by reward processing dysfunctions, such as substance dependence. ${ }^{15,16}$ The $\mathrm{CN}$ is also a dopaminergic structure and is part 
of the nigrostriatal circuit. There is evidence of alterations in the $\mathrm{CN}$ in other disorders characterized by impulsivity and lack of inhibitory control, such as attention deficit hyperactivity disorder. ${ }^{17} \mathrm{~A}$ previous study by Marsh et al. ${ }^{3}$ in 2009 showed that in a task of selective attention, patients with $\mathrm{BN}$ made more errors and failed to activate the OFC and the anterior cingulate cortex (ACC), which are regions involved in self-regulatory control. These findings are consistent with the presence of selfregulatory deficits in patients with $\mathrm{BN}$ and the relationship with orbitofrontostriatal abnormalities. In accordance, frontal-striatal regions have been reported to be altered in $\mathrm{BN} .{ }^{18,19} \mathrm{~A}$ recent study by Schäfer et al. (2010) found that individuals with BN had increased gray matter (GM) volume in the left and right medial OFC and the left and right VST compared with individuals with binge eating disorder (BED) and that the BMI and purging severity were correlated with the striatal GM volume. ${ }^{18}$ No differences were observed in the global GM or global and regional white matter (WM) and cerebral spinal fluid (CSF) volume. By contrast, a recent VBM (voxel-based-morphometry) study ${ }^{19}$ reported regional GM reduction in the $\mathrm{CN}$ in $\mathrm{BN}$ compared to anorexia nervosa (AN) and healthy controls (HC). However, other authors found no evidence for structural alterations either on the GM or WM volume of patients with $\mathrm{BN} .^{20-22}$

Inconsistent findings across structural neuroimaging studies may be associated with clinical sample heterogeneity, different analysis techniques, and the use of different segmentation methods. We believe that the use of manual segmentation methods in a Region of Interest (ROI) approach can help identify more subtle volumetric alterations associated with specific neurocognitive alterations (e.g., inhibitory control). Manual segmentation has the advantage of providing a direct volume analysis, being better able to adapt to the individual variability in the brain's shape and volume. ${ }^{23,24}$ Additionally, when the ROI under study has no visible anatomical boundaries, manual tracing methods are strongly advised.

The objective of this study was to compare individuals with a $\mathrm{BN}$ diagnosis with healthy matched controls, using a ROI approach. Based on previous research, ${ }^{18}$ and on the possible mediator role of VST in the processing of the hedonic value of food, we hypothesized that patients diagnosed with BN will show an increased NAc volume when compared to HC. Furthermore and taking into account recent findings of $\mathrm{CN}$ volume reduction in $\mathrm{BN},{ }^{19}$ we hypothesized that patients with $\mathrm{BN}$ will exhibit morphologic alterations in the $\mathrm{CN}$, potentially related to difficulties in inhibitory control.

\section{Method}

\section{Participants}

Forty-one female subjects, 21 diagnosed with $\mathrm{BN}$ and $20 \mathrm{HC}$, comparable as to age, sex, handedness, and educational level, participated. Patients were recruited in the Outpatient Clinic of Eating Disorders Unit in Hospital de S. João EPE, Porto. Clinical diagnosis of BN was done according to DSM-IV criteria and confirmed by two clinical psychiatrists, one of them an expert in Eating Disorders. The exclusion criteria for MRI acquisition were: pregnancy, lactation, or planning to become pregnant in the next 18 months; postpartum ( $<1$ year); claustrophobia; metal implants or non-removable metal dental retainers, and pacemakers incompatible with the MR environment; history of severe head injury; and neurological disorder. Specific exclusion criteria for this study were: psychotropic medication other than antidepressants and anxiolytics; alcohol consumption ( $>3$ drinks/ day); substance abuse disorder; suicidal ideation; Axis I mental disorder other than ED (since affective and obsessive-compulsive symptoms are integral parts of ED); psychotic disorder or hospitalization for psychiatric illness within one year prior to the beginning of the study; history of significant health problems, such as endocrine disorder (including diabetes), cancer, heart, kidney, thyroid, gastrointestinal, or liver disease; previous abdominal or brain surgery.

From the initial sample of 23 patients with $\mathrm{BN}$ and 22 $\mathrm{HC}$ that were assessed, two patients with $\mathrm{BN}$ were excluded: one due to incomplete neuroimaging data and one due to movement artefacts) and two healthy subjects were excluded (one due to claustrophobia and one due to visual impairment). Thus there were 21 patients with $\mathrm{BN}$ and 20 normal controls who completed the study.

The two groups did not differ as to mean BMI. Participants were instructed to fast for $3 \mathrm{~h}$ before undergoing the MRI, to ensure that they had undergone comparable periods of fasting before the acquisition. Compliance was assessed by self-report. Co-morbid psychopathology was assessed, including general symptomatic distress, impulsivity, past history of substance abuse, and ED symptoms, and the scores on the different symptom measures are summarized in Table 1.

Handedness was assessed by a semi-structured interview in which the participants were asked to indicate their hand preferences in activities such as drawing; using the knife without fork; using the broom (upper hand), or opening the lid box. Handedness was similar between the groups, with one left-handed participant in 
TABLE 1. Demographic and clinical characteristics for patients with bulimia nervosa and healthy controls (HC)

\begin{tabular}{lccc}
\hline Variable & $\mathrm{HC}, n=20$ & $\mathrm{BP}, n=21$ & $\begin{array}{c}\text { Total sample, } \\
N=41\end{array}$ \\
\hline Age (years) & $30.90(8.79)$ & $31.57(8.27)$ & $31.24(8.42)$ \\
$\begin{array}{l}\text { Education } \\
\quad \text { school years) }\end{array}$ & $15.80(3.22)$ & $13(4.51)$ & $14.37(4.13)$ \\
Weight (Kg) & $58.95(8.61)$ & $58.62(10.97)$ & $58.78(9.77)$ \\
Height (m) & $1.64(.070)$ & $1.64(.049)$ & $1.64(.060)$ \\
BMI (kg) & $22.11(3.20)$ & $21.39(2.36)$ & $21.74(2.79)$ \\
Duration of & - & $12.10(10.16)$ & $54.49(44.56)$ \\
$\quad$ illness (years) & & & \\
EDE_Q Total & $0.552(.74)$ & $4.05(1.30)$ & $2.34(2.06)$ \\
BIS-11Total & $38.55(6.32)$ & $49.62(24.48)$ & $44.22(18.71)$ \\
OQ45 Total & $31.25(14.87)$ & $191.7(272.45)$ & $113.41(209.3)$ \\
\hline
\end{tabular}

Notes: BMI, body mass index; EDE, Eating Disorders Questionnaire; BIS, Barret Impulsivity Scale; 0Q45, Outcome Questionnaire.

each group. In terms of medications, 15 patients were taking psychotropic medication: 6 only antidepressants, 2 only anxiolytics, 7 both antidepressant and anxiolytics.

The project was approved by the IRB of the University in which the study was conducted and the local Hospital in which the data were collected. Written informed consent was obtained from all participants after they received a complete description of the study.

\section{Clinical Measures}

The clinical assessment of the participants consisted of a clinical interview and a battery of instruments:

Outcome Questionnaire 45 (OQ45) - The OQ-45 is a 45-item self-report measure that takes about $5 \mathrm{~min}$ to complete and assesses mentally healthy functioning and quality of life. It is composed of three subscales: (a) subjective psychopathology, (b) interpersonal relationships, and (c) social role functioning. Each item on the OQ-45 was rated on a 5 -point scale $(0=$ never, $1=$ rarely, $2=$ sometimes, $3=$ frequently, or $4=$ almost always), yielding a range of possible scores from 0 to 180, with higher values indicating poorer functioning. The Portuguese version of the scale was used and has good psychometric properties. $^{25}$

- Barratt Impulsiveness Scale (BIS-11)—The BIS is a 30item self-report measure that assesses impulsivity. It has three subscales: motor impulsivity, attentional impulsivity, and lack of planning. Each item is rated on a 4-point scale, yielding a range of total possible scores from 30 to 120 , with higher values indicating more impulsivity. The European Portuguese version of the BIS-11 was adapted from the Brazilian Portuguese version of the BIS- $11 .^{26}$

- Eating Disorders Examination-Questionnaire (EDEQ) -A semi-structured interview with 36 questions organized into 4 subscales: shape concern; weight concern, restriction, and food concern-that refers to behaviors, attitudes, or feelings that occurred in the previous 28 days. Each item is rated from 0 to 6 , indi- cating the number of days during which the referred behaviors, attitudes, or feelings occurred. The Portuguese version of the EDE-Q was adapted by Machado et al. in $2013 .^{27}$

\section{Magnetic Resonance Imaging Acquisition}

The structural images were acquired using a SIEMENS Magnetom TrioTimon scanner, 3 Tesla scanner MAGNETOM Trio A Tim (Siemens, Erlangen, Germany) equipped with a 12-channel head matrix coil. The following were the acquisition parameters: flip angle $=9^{\circ}$, repetition time $=2,300 \mathrm{~ms}$, echo time $=2.98 \mathrm{~ms}$, inversion time $=$ $900 \mathrm{~ms}$, Field of View $(\mathrm{FoV})=240 \mathrm{~mm} \times 256 \mathrm{~mm}$, acquisition matrix $=256 \times 240 \mathrm{~mm}$; phase-encoding step$\mathrm{s}=240 \mathrm{~mm}$ and voxel size: $1 \times 1 \times 1.2$.

\section{Post-processing}

After the acquisition, the images were reconstructed and analyzed using FreeSurfer (http://surfer.nmr.mgh. harvard.edu/), a widely used software, and a welldeveloped application. ${ }^{28,29}$ A manual verification of the reconstructed images consisted of: verification of the coregistration in the Tailarach template, which is based on the distance between the anterior (AC) and posterior commissure (PC); verification of skull removal from the images, in order to correct for mistakes of brain tissue and verification of the limits of the cortical and subcortical areas. ${ }^{30}$ After this verification, we obtained the processed images, allowing for the application of a consistent protocol of GM identification in the ROI. The intracranial total volume (ITV) was calculated using the statistics from Free Surfer.

\section{Regions of Interest}

For ROI tracing, we employed 3D-Slicer, an imageediting tool developed at BWH, Psychiatry Neuroimaging Lab and MIT (http://www.slicer.org). The volume was computed as the sum of all the voxels included in the specific ROIs under study. We followed the protocol of manual segmentation proposed by Levitt et al., ${ }^{31}$ with the following anatomical landmarks of the NAc and CN.

At the level of the anterior commissure-posterior commissure (AC-PC) plane, in the axial view (Fig. 1A), we drew a large polygon which appears as a line in the coronal view, which was used as a landmark for tracing the NAc (Fig. 1B). The most posterior slice of the NAc was then located which was the first slice where the AC no longer protrudes toward the midline of the brain, and where GM of the NAc still clearly connects the caudate and putamen, and the WM external capsule is present inferiorly (Fig. 1C). To delineate the NAc, we drew two intersecting lines, the first one skimming the superior surfaces of the caudate and putamen, the second, drawn along the plane formed by the IC-putamen 
FIGURE 1. Representation of the polygon drawn on axial view at the level of AC-PC plane (A); in (B) we see the coronal view of this polygon (a line) and in (C) we see the most posterior coronal slice of the NAc in coronal view. [Color figure can be viewed in the online issue, which is available at wileyonlinelibrary.com.]

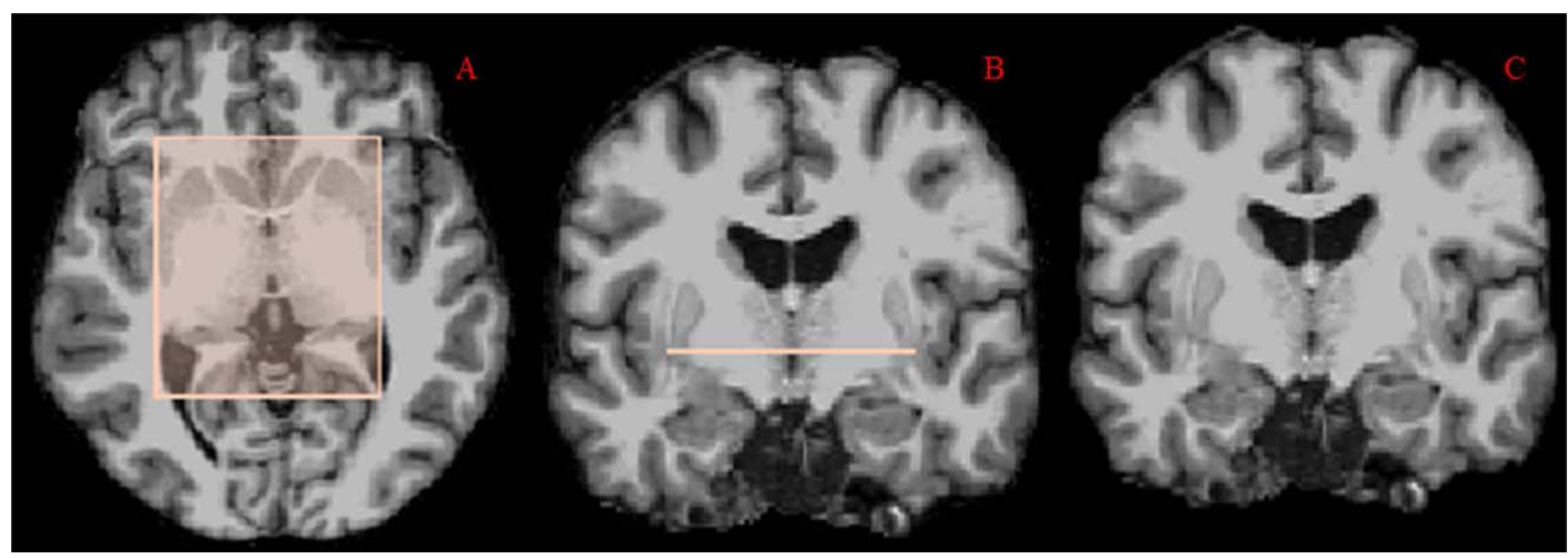

FIGURE 2. Methods to delineate the NAc. A: Shows a X formed by two intersecting lines at the top of the putamen; (B,C) show landmarks to draw the oblique line that limits the NAc superiorly; (D) shows the more anterior part of the NAc. [Color figure can be viewed in the online issue, which is available at wileyonlinelibrary.com.]

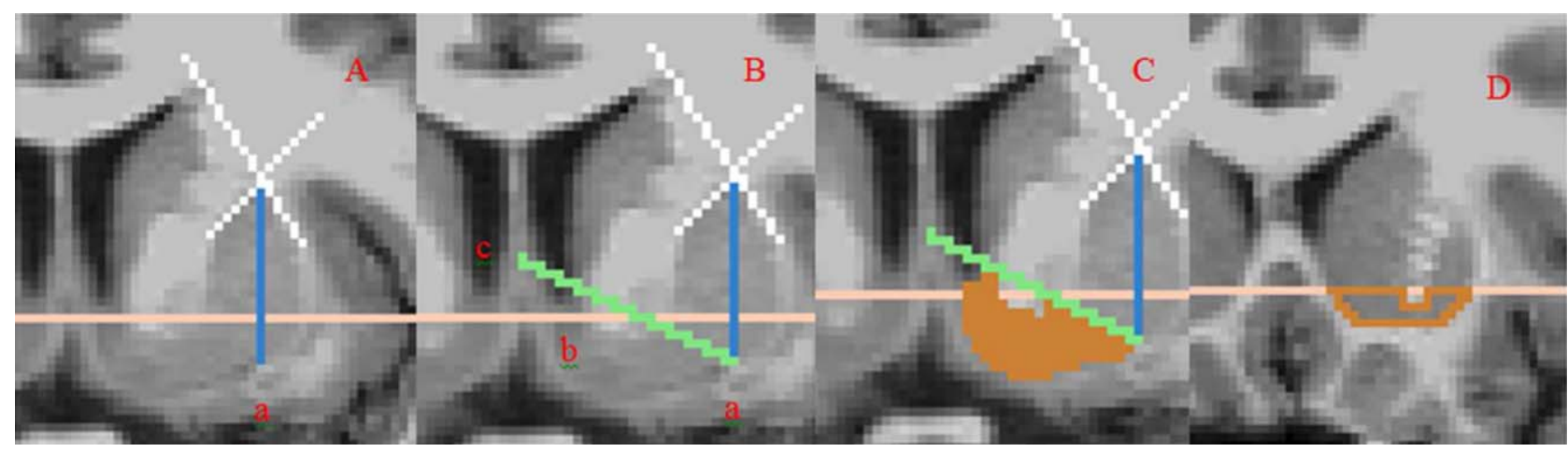

border intersecting the first (forming an "X"). A vertical line from the intersection of this " $\mathrm{X}$ " (the uppermost point of the putamen) was drawn to the ventrolateral border of the putamen, marking Point $a$ (Fig. 2A). To define the dorsal boundary of the NAc, the midpoint between the medial edge of the caudate and lateral edge of the putamen at the level of AC-PC plane was marked (Point $b$ ). An oblique line was then drawn connecting Points $a$ and $b$ and extending superiorly and medially to the medial border of the caudate (Point $c$; Fig. 2B). This oblique line served as the dorsal boundary of the NAc. Medial and ventral boundaries were formed by the WM external capsule separating the VST from the basal forebrain. For the most anterior slices, the vertical dropped line does not pass through the AC-PC, and for these slices, the AC-PC planes form the dorsal boundary. The NAc was drawn anteriorly until it was no longer present at or below the level of the AC-PC plane (Fig. 2D). The caudate nuclei were measured bilaterally, using three orthogonal planes, in all slices in which they appeared. The head, body, and tail portions of the caudate were included up to the point where the tail curved ventrally to border the lateral aspect of the atrium of the lateral ventricles (Figs. 3 and 4).

\section{Reliability Analysis}

Two independent raters, using the same protocol of segmentation of the ROIs, segmented the images of 12 cases that correspond to $30 \%$ of the sample. Raters were two researchers, one postdoctoral researcher and a master level researcher, both clinical psychologists. Raters measured all slices in which the ROIs were present and were blind to the diagnoses of the cases. Intraclass correlation (inter-rater reliability) was high for both ROIs. 
FIGURE 3. Methods to segment the CN. [Color figure can be viewed in the online issue, which is available at wileyonlinelibrary.com.]

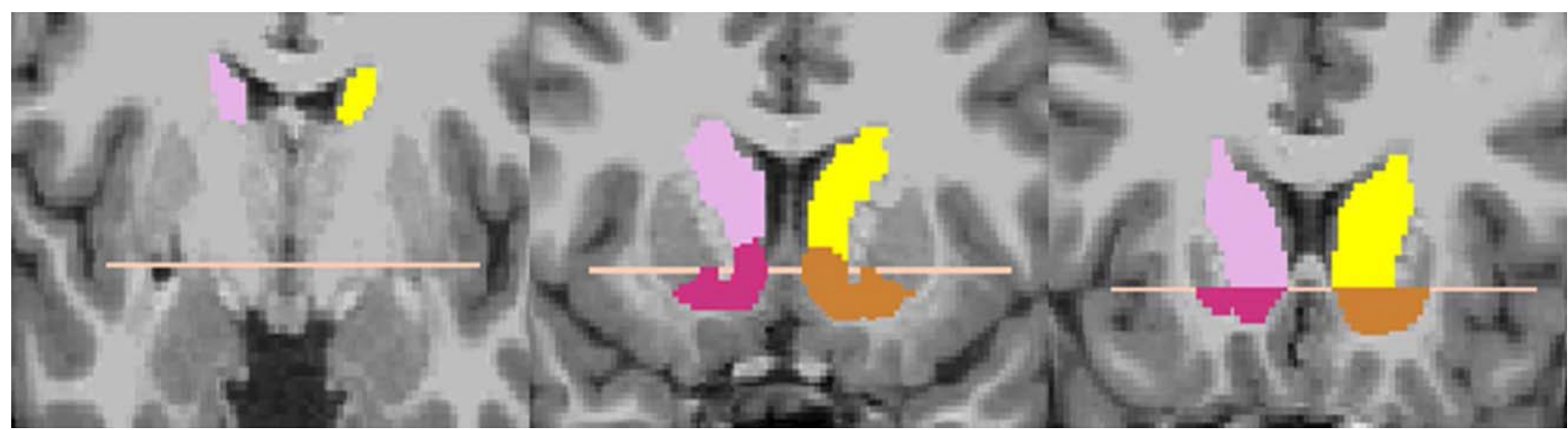

FIGURE 4. 3D reconstruction of the NAC and CN after the total manual segmentation of both structures. [Color figure can be viewed in the online issue, which is available at wileyonlinelibrary.com.]

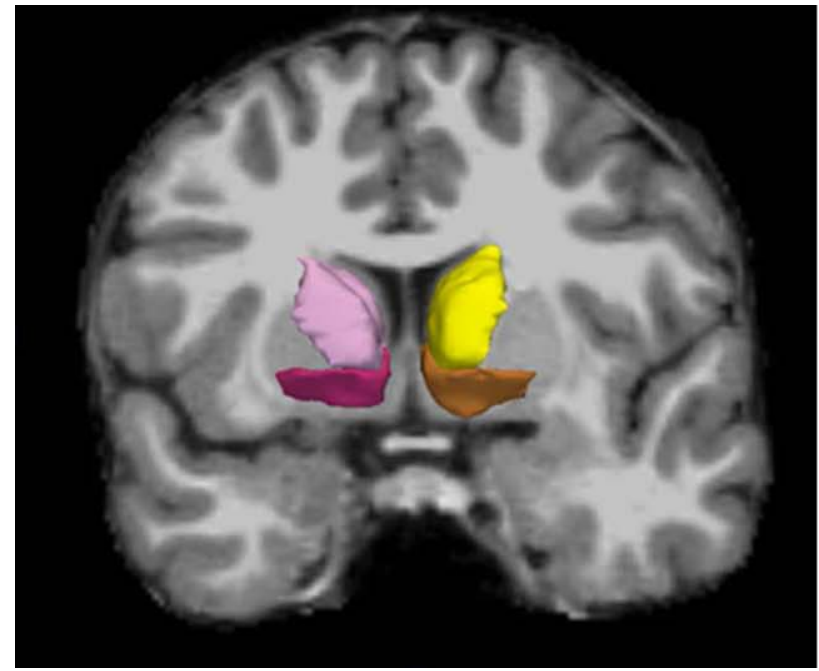

For the right NAc, $(r=.96)$; for the left NAc $(r=.935)$. For the right $\mathrm{CN}(r=.89)$; for the left $\mathrm{CN}(r=.91)$.

\section{Statistical Analysis}

Data analysis revealed that for both the NAc and CN volumes, the normality (Kolmogorov-Smirnov, $p>.05$; Shapiro-Wilk, $p>.05$ ) and variance homogeneity (Levene Statistic, $p>.05$ ) assumptions were met. In order to correct for head size variation, for each ROI we calculated the relative volume by dividing absolute volumes by total intracranial volume and multiplying by 100 . Statistical analyses for volume measures were performed only on relative brain volumes. The relative NAc and CN were submitted to a mixed model ANOVA with diagnostic group (BN vs. $\mathrm{HC}$ ) as the "between-subjects" factor, and hemisphere (left vs. right) as the "within-subjects" factors. To test for possible associations between the volume

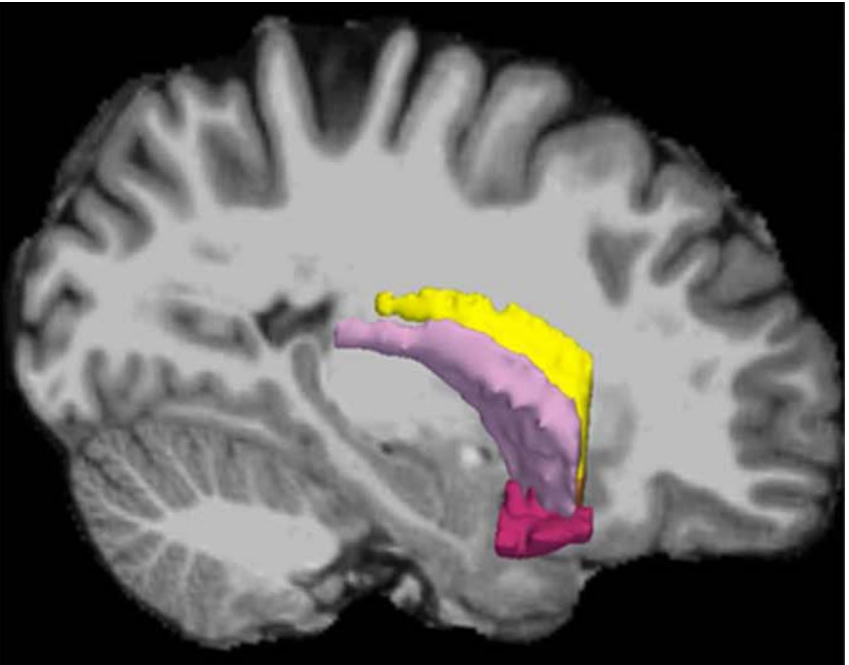

of the NAc and CN and clinical and demographic measures, Pearson's correlation tests were performed.

\section{Results}

The overall sample had a mean of 14.37 years of education $(\mathrm{SD}=4.13)$. The mean age for patients with $\mathrm{BN}$ was $31.51(\mathrm{SD}=8.27)$ and that for normal controls was 30.9 ( $\mathrm{SD}=8.79)$. For the $\mathrm{BN}$ group, the mean weight was $58.62 \mathrm{~kg}(\mathrm{SD}=10.97)$, mean BMI $21.39(\mathrm{SD}=2.36)$, and mean duration of the disorder, $12.10(\mathrm{SD}=10.16)$ years (Table $\mathbf{1})$.

\section{Total Intracranial Volume}

The descriptive values for the ITV, NAc, and CN volume of participants with $\mathrm{BN}$ and $\mathrm{HC}$ are shown in Table 2. For the ITV we found marginally 
TABLE 2. Summary of the volumes

\begin{tabular}{|c|c|c|c|c|c|c|c|c|c|}
\hline \multicolumn{10}{|c|}{ Volume Mean (SD) } \\
\hline & \multirow[b]{2}{*}{ ITV (ml) } & \multicolumn{2}{|c|}{ NAc Absolute (ml) } & \multicolumn{2}{|c|}{ NAc Relative (ml) } & \multicolumn{2}{|c|}{ CN Absolute (ml) } & \multicolumn{2}{|c|}{ CN Relative (ml) } \\
\hline & & Left & Right & Left & Right & Left & Right & Left & Right \\
\hline $\mathrm{BN}(n=21)$ & $1316.62(86.80)$ & $1.02(0.41)$ & $0.94(0.46)$ & $0.076(0.03)$ & $0.07(0.03)$ & $2.78(0.47)$ & $3.01(0.47)$ & $0.21(0.03)$ & $0.22(0.03)$ \\
\hline $\mathrm{HC}(n=20)$ & 1259.17 (117.57) & $0.97(0.41)$ & $0.99(0.42)$ & $0.076(0.03)$ & $0.07(0.03)$ & $2.95(0.27)$ & $3.08(0.24)$ & $0.23(0.02)$ & $0.24(0.02)$ \\
\hline Total $(N=41)$ & $1288.60(105.72)$ & $0.99(0.40)$ & $0.94(0.43)$ & $0.076(0.03)$ & $0.07(0.03)$ & $2.86(0.39)$ & $3.05(0.37)$ & $0.22(0.03)$ & $0.23(0.03)$ \\
\hline
\end{tabular}

Notes: ITV, intracranial total volume; BN, bulimia nervosa; HC, healthy controls; NAC, nucleus accumbens; CN, caudate nucleus.

FIGURE 5. Mean volumes of the NAC in patients with BN and $\mathrm{HC}$ (healthy control) groups. Error bars represent standard deviations.

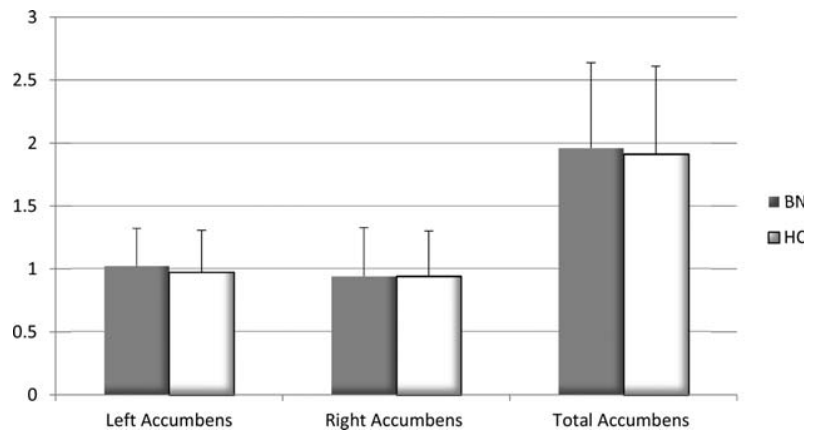

significant differences between $\mathrm{BN}$ and $\mathrm{HC}$ : $t(39)=-1.79, p=.08$, based on ITV, $\mathrm{M}=1,316.6$; $\mathrm{SD}=86.81$ for $\mathrm{BN}$, as compared to $\mathrm{HC}$, $\mathrm{M}=1,259.2 ; \mathrm{SD}=117.57^{\mathrm{a}}$. Accordingly, as already mentioned, the statistical tests of differences for NAc and CN were performed on relative brain volumes.

\section{Volume of the NAC}

No significant group differences were observed regarding the relative volume of the NAc in participants with $\mathrm{BN}$ versus $\mathrm{HC}, F(1,39)=0.02, p=.88$. We also did not find an hemisphere effect, $F$ $(1,39)=2.68, p=.109$, or an interaction effect, $F$ $(1,39)=0.468, p=.498$ (Fig. 5).

\section{CN Volume}

We found a significant effect between groups for the relative volume of the $\mathrm{CN}$ in $\mathrm{BN}$ compared with HCs: $F(1,39)=5.26, p=.002$. Post hoc $t$-tests

\footnotetext{
${ }^{\mathrm{a}}$ To test for any uncontrolled influences of laterality upon our findings, we re-ran tests for group differences on ITV, left CN volume, right CN volume, and total CN volume, but this time excluding the two left-handed participants from analyses. Results of the latter analyses were comparable to those obtained in the full sample, suggesting that handedness had no substantive effect. We report results from the full-sample analyses $(n=41)$ in the text.
}

FIGURE 6. Mean volumes of the $\mathrm{CN}$ in patients with $\mathrm{BN}$ and $\mathrm{HC}$ (healthy control) groups. Error bars represent standard deviations.

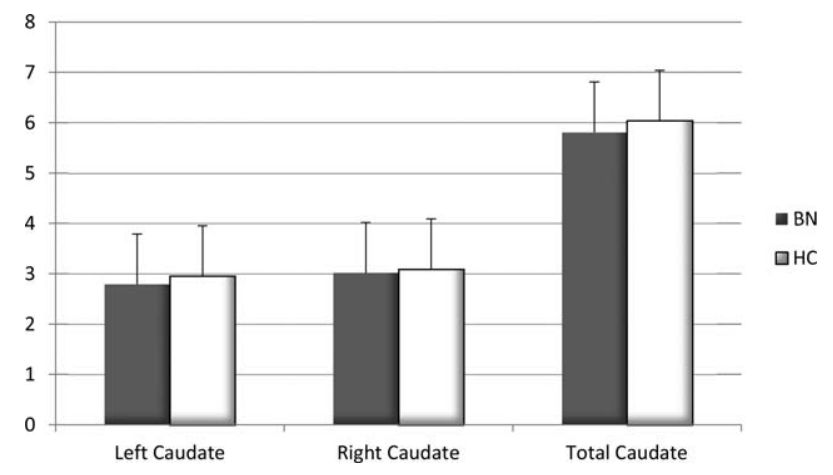

showed a decreased volume of the left $\mathrm{CN}$ volume $(t(39)=2.628, p=.001)$, the right $\mathrm{CN}$ volume $(t$ $(39)=1.82, p=.007)$ and the total $\mathrm{CN}$ volume $(t$ $(39)=2.27, p=.002)$ in the BN group when compared to the HC group (Fig. 6).

\section{Correlations Between Volumetric Data of the CN and Clinical Measures}

We found a marginally significant negative association between the subscale of attention impulsivity of the BIS and the left $(r=-.389, p=.08)$, right $(r=-.386, p=.08)$, and total $(r=-.395$, $p=.08) \mathrm{CN}$ volume. Thus, a higher score in this impulsivity scale tended to be associated with a smaller CN volume. Also the total score of the BIS tended to be negatively associated with the left $(r=-.380, p=.09)$ and total $(r=-.378, p=.09)$ $\mathrm{CN}$ volume.

\section{Discussion}

The main goal of this study was to assess morphologic alterations in the NAc and $\mathrm{CN}$ in patients diagnosed with BN. Our results provided empirical evidence to support one of these hypotheses. We found evidence for a volume reduction in the $\mathrm{CN}$ in those with $\mathrm{BN}$ when compared to HC. However, 
no volumetric significant differences in NAc were found between the groups.

The finding of a $\mathrm{CN}$ volumetric reduction in $\mathrm{BN}$ is consistent with evidence of difficulties in the inhibitory control and the presence of impulsive behaviors in $\mathrm{BN}$ and with a recent study by Amianto et al. in 2013, ${ }^{19}$ that reported a GM reduction in the $\mathrm{CN}$ in $\mathrm{BN}$ compared to $\mathrm{AN}$ and controls. The authors argued that the alterations in the CN could be associated with the binge eating behavior. Previous studies have supported the presence of self-regulatory deficits in $\mathrm{BN}$, manifested in the impulsive behavior of food intake. ${ }^{3,32,33}$ The dysregulation of frontostriatal circuits maybe a core characteristic of eating disorders. ${ }^{3}$ Our results also showed a trend toward association between the volume of the $\mathrm{CN}$ and scores on impulsivity assessed by the BIS.

We hypothesize that impulsivity may be associated with a dysregulation of the frontostriatal circuits, preventing the patients from adequately controlling their eating behavior, which can contribute to their binge eating episodes. The $\mathrm{CN}$ projects to the orbitofrontal cortex (OBF), and anatomical alterations in structures within these frontostriatal circuits may result in behaviors that are characterized by a deficit in inhibitory control. Reductions of the CN volume may also be associated with altered functional activation underlying the impaired inhibitory control present in these patients. ${ }^{3,9}$ To test this hypothesis, however, additional studies would be needed to analyze the functional activation of the $\mathrm{CN}$ using inhibitory control tasks.

The NAc has an important role in the processing of rewarding and pleasant stimuli, such as palatable food. ${ }^{18,34,35}$ Therefore, we might anticipate alterations in this brain region in a disorder that is characterized by the episodic intake of large amounts of high calorie food. The absence of volumetric alterations in NAc in BN appears to contrast with previous research by Schafer et al. ${ }^{18}$ that reported an enlargement of the VST in those with $\mathrm{BN}$. The authors suggested that the increased volume of the NAc could be related not only with alterations in the processing of the hedonic value of food, but also to the chronic behaviors that lead the patient to obtain a reward.

In disorders characterized by alterations in the reward processing of stimuli (such as substance dependence), volumetric alterations in brain regions involved in reward circuits have also been found ${ }^{15,17}$ However, in the case of drug abuse, those alterations may be related to the neurotoxic effects of the drugs taken, ${ }^{36-39}$ a factor that is specific to drug-addicted patients and not present in our sample of patients with BN. In the case of BN, observed morphological alterations of striatal structures involved in dopaminergic circuits are more likely to be related to alterations in brain function that influence reward and goal-directed behavior.

The absence of significant volumetric differences in the NAc between BN and HC that we found suggests that the NAc at the volumetric level may not be a neuroanatomical marker of BN. The absence of volumetric alterations in the NAc however, does not mean that this region is functionally intact. Our findings about the NAc are consistent with those reported by previous studies. ${ }^{20-22}$ Joos et al. ${ }^{22}$ who compared 17 women with $\mathrm{BN}$ and 18 matched controls, using VBM did not find significant betweengroup differences in whole and regional brain GM, WM, or CSF volume fractions.

The differences across studies may relate to methodological factors, as more recent studies use VBM, an automated rather than a manual segmentation approach that tackles GM density and is not a direct measure of volume. Other likely explanations to these inconsistencies in neuroimaging studies are: diverse clinical sample characteristics, symptom severity, illness duration, comorbid psychopathology, and a previous history of AN.

Based on our results, we may speculate about the existence of a volumetric dissociation between ventral (NAc) and dorsal striatum $(\mathrm{CN})$ regions in $\mathrm{BN}$. One can hypothesize that $\mathrm{BN}$ is predominantly a disorder of impulse control, independently of the reward value or the pleasure provided by food. We also suggest that the main brain alteration of these patients may be at the level of the mesostriatal pathway (substantia nigra -----CN) rather than mesolimbic pathway (VTA ------NA). Future functional connectivity studies may help to clarify the hypotheses of dissociation between dorsal and ventral basal ganglia functioning in $\mathrm{BN}$.

The present study has some limitations. First, only female participants were studied, preventing the possibility of any generalizations to male patients. Second, although we found volumetric alterations in the dorsal striatum, a region associated with behavioral self-regulation, it remains unclear whether these alterations are a cause or a consequence of the disorder. Cross-sectional imaging differences are correlational, and we cannot infer causality from these results. Future longitudinal studies can further explore whether these alterations are acute-phase abnormalities, 
neurobiological risk factors, or potential biomarkers of the disorder.

Moreover future studies should explore structural and functional alterations of other ROIs potentially associated with the psychopathology and pathophysiology of bulimia. From the ROIs to be analyzed in future studies we highlight the cortical areas involved in the fronto-striatal circuits of inhibitory control such as the dorsolateral prefrontal cortex and OBF cortex that has shown to have a role in processing of food cues ${ }^{40-42}$; the ACC and insula both involved in selective attention and introception $^{5,42}$; and the fusiform gyrus that is involved in the processing of body image. ${ }^{43-45}$

Our study supports the evidence of a decreased GM volume in the $\mathrm{CN}$ in BN. Furthermore, the absence of volumetric alterations in the NAc points to a possible volumetric dissociation between dorsal and VST regions in BN. A better understanding of the association between imaging brain alterations and neurocognitive features in BN may lead to novel treatments and interventions, as well as have diagnostic and prognostic value.

The authors acknowledge the support of Dr. António Bastos Leite and the Department of Medical Imaging, Faculty of Medicine, University of Porto. The magnetic resonance imaging scanning sessions were kindly granted (free of charge, for the external Institutions) by means of an agreement between the Department of Medical Imaging (Faculty of Medicine, University of Porto) and the Departments of Radiology and Psychiatry (Hospital de São João, Porto) aimed at accomplishing several research projects on neuroimaging in psychiatry underway.

\section{References}

1. Diagnostic and Statistical Manual of Mental Disorders DSM-5, 5th ed. Arlington, VA: American Psychiatric Publishing, 1994.

2. Harrison A, O'Brien N, Lopez C, Treasure J. Sensitivity to reward and punishment in eating disorders. Psychitry Res 2010;177:1-11.

3. Marsh R, Steinglass JE, Gerber AJ, O'Leary KG, Wang Z, Murphy D, et al. Deficient activity in the neural systems that mediate self-regulatory control in bulimia nervosa. Arch Gen Psychiatry 2009;66: 51-63.

4. Rosval L, Steiger H, Bruce K, Israël M, Richardson J, Aubut M. Impulsivity in woman with eating disorders: Problem of response inhibition, planning, or attention? Int J Eat Disord 2006;39:590-593.

5. Wagner A, Greer P, Bailer UF, Frank GK, Henry SE, Putnam K, et al. Normal brain tissue volumes after longterm recovery in anorexia and bulimia nervosa. Biol Psychiatry 2006;59:291-293.

6. Alexander GE, DeLong MR, Strick PL. Parallel organization of functionally segregated circuits linking basal ganglia and cortex. Annu Rev Neurosci 1986;9:357-381.

7. Alexander GE, Crutcher MD, DeLong MR. Basal ganglia-thalamocortical circuits: parallel substracts for motor, oculomotor, "prefrontal" and "limbic" functions. Prog Brain Res 1990;85:119-146.
8. Gray A, McNaughton N. The Neuropsychology of Anxiety: An Enquiry into the Functions of the Septo-hippocampal System, 2nd ed. Oxford: Oxford University Press, 2000.

9. Berridge KC. 'Liking' and 'wanting' food rewards: Brain substrates and roles in eating disorders. Physiol Behav 2009;97:537-550.

10. Bohon C, Stice E. Negative affect and neural response to palatable food intake in bulimia nervosa. Appetite 2012;58:964-970.

11. DiLeone RJ. The influence of leptin on the dopamine system and implications for ingestive behaviour. Int J Obes 2009;33:25-29.

12. Fulton S, Pissios $P$, Manchon RP, Stiles L, Frank L, et al. Leptin regulation of the messoaccumbens dopamine pathway. Neuron 2006;51:811-822.

13. Stahl SM. Chapter 9: Drug treatments for obsessive-compulsive disorder, panic disorder, and phobic disorders. In: Stahl SM, ed. Essential Psychopharmacology: Neuroscientific Basis and Pratical Applications. New York, NY: Cambridge University Press, 2000, pp. 335-364.

14. Nicola SM. The nucleus accumbens as part of a basal ganglia action selection circuit. Psychopharmacology 2007;191:521-550.

15. Franklin TR, Acton PD, Maldjian JA, Gray JD, Croft JR, Dackis CA, et al. Decreased grey matter volume in the insular, orbitofrontal, cingulate, and temporal cortices of cocaine patients. Biol Psychiatry 2002;51:134-142.

16. Tanabe J, Tregellas JR, Dalwani M, Thompson L, Owens E, Crowley T, et al. Medial orbitofrontal cortex grey matter is reduced in abstinent substance dependent individuals. Biol Psychiatry 2009;65:1160-1164.

17. Castellanos FX, Giedd JN, Eckburg P, Marsh WL, Vaituzis AC, Kaysen D, et al. Quantitative morphology of the caudate nucleus in attention deficit hyperactivity disorder. Am J Psychiatry 1994;151:1791-1796.

18. Shafer A, Vaitl D, Schienle A. Regional grey matter abnormalities in bulimia nervosa and binge-eating disorder. Neurolmage 2010;50:639-643.

19. Amianto F, Caroppo P, D’Agata F, Spalatro A, Lavagnino L, Caglio M, et al. Brain volumetric abnormalities in patients with anorexia and bulimia nervosa: A voxel-based morphometry study. Psychiatry Res 2013;213: 210-216.

20. Lankenau H, Swigar ME, Bhimani S, Luchins D, Quinlan DM. Cranial CT scans in eating disorder patients and controls. Compr Psychiatry 1985;26: 136-147.

21. Hussain MM, Black KJ, Doraiswamy PM, Shah SA, Rockwell WJ, Ellinwood $\mathrm{EH}$, et al. Subcortical brain anatomy in anorexia and bulimia. Soc Biol Psychiatry 1992;31:735-738.

22. Joos A, Kloppel S, Hartmann A, Glauche V, Tuscher O, Perlov E, et al. Voxelbased morphometry in eating disorders: Correlation of psychopathology with grey matter volume. Psychiatry Res 2010;182:146-151.

23. Yushkevich PA, Piven J, Hazlett HC, Smith RG, Ho S, Gee JC, et al. User-guided 3D active contour segmentation of anatomical structures: Significantly improved efficiency and reliability. Neurolmage 2006;31:1116-1128.

24. Bokde AL, Teipel SJ, Schwarz R, Leinsinger G, Buerger K, Moeller T, et al. Reliable manual segmentation of the frontal, parietal, temporal, and occipital lobes on magnetic resonance images of healthy subjects. Brain Res Brain Res Protoc 2005; 14:135-145.

25. Machado PP, Fassnacht D. The Outcome Questionnaire (0Q-45.2) in a Portuguese population: Psychometric properties, norms and confirmatory factor analysis. Manuscript submitted for publication.

26. Malloy-Dinez L, Mattos P, Leite WB, Abreu N, Coutinho G, de Paula JJ, et al. Translation and cultural adaptation of Barratt Impulsiveness Scale (BIS-11) for administration in Brazilian adults. Jornal Brasileiro de Psiquiatria 2010; 59:99-105.

27. Machado PP, Machado BC, Martins C, Gonçalves S. Eating Disorder Examination Questionnaire (EDE-Q): Psychometric properties and norms for the portuguese population. Manuscript submitted for publication.

28. Reuter M, Schmansky NJ, Rosas HD, Fischl B. Within-subject template estimation for unbiased longitudinal image analysis. Neurolmage 2012;57:19-21.

29. Hodneland E, Ystad M, Haasz J, Munthe-Kaas A, Lundervold A. Automated approaches for analysis of multimodal MRI acquisition in a study of cognitive aging. Comput Methods Programs Biomed 2012;106:328-341.

30. Fischl B, Salat DH, van der Kouwe AJ, Makris N, Segonne F, Quinn BT, et al. Sequence-independent segmentation of magnetic resonance images. Neurolmage 2004;23:69-84. 
31. Levit JJ, Rosow LK, Nestor PG, Pelavin PE, Swisher TM, McCarley RW, et al. A volumetric MRI study of limbic, associative and sensorimotor striatal subregions in schizophrenia. Schizophrenia Res 2013;23:24-33.

32. Fahy T, Eisler I. Impulsivity and eating disorders. Br J Psychiatry 1993;162: 193-197.

33. Marsh R, Horga G, Wang Z, Wang P, Klahr KW, Berner LA, et al. An fMRI study of self-regulatory control and conflict resolution in adolescents with bulimia nervosa. Am J Psychiatry 2011;168:1210-1220.

34. Diekhof EK, Falkai P, Gruber O. Functional neuroimaging of reward processing and decision-making: A review of aberrant motivational and affective processing in addiction and mood disorders. Brain Res Rev 2008;59:164-184.

35. O'Doherty JP, Deichmann R, Crichley HD, Dolan RJ. Neural responses during anticipation of a primary taste reward. Neuron 2002;33:815-826.

36. Andersen SN, Skullerud K. Hypoxic/ischaemic brain damage, especially pallidal lesions, in heroin addicts. Forensic Sci Int 1999;102:51-59.

37. Danos P, Kasper S, Grunwald F, Klemm E, Krappel C, Broich K, et al. Pathological regional cerebral blood flow in opiate-dependent patients during withdrawal: A HMPAO-SPECT study. Neuropsychobiology 1998;37:194-199.
38. Rose JS, Branchey M, Buydens-Branchey L, Stapleton JM, Chasten K, Werrell $A$, et al. Cerebral perfusion in early and late opiate withdrawal: A technetium-99m-HMPAO SPECT study. Psychiatry Res 1996;67:39-47.

39. Shalev U, Grimm JW, Shaham Y: Neurobiology of relapse to heroin and cocaine seeking: A review. Pharmacol Rev 2002;54:1-42.

40. Karhunen LJ, Vanninen EJ, Kuikka JT, Lappalainen RI, Tiihonen J, Uusitupa MJ. Regional cerebral blood flow during exposure to food in obese binge eating women. Psychiatry Res Neuroimage 2000;99:29-42.

41. Uher R, Murphy T, Brammer MJ, Dalgleish T, Phillips ML, Andrew CM, et al. Medial prefrontal cortex activity associated with symptom provocation in eating disorders. Am J Psychiatry 2004;161:1238-1246.

42. Schienle A, Schäfer A, Hermann A, Vaitl D. Binge-eating disorder: Reward sensitivity and brain activation to images of food. Biol Psychiatry 2009;65: 654-661.

43. Peelen MV, Downing PE. Selectivity for the human body in the fusiform gyrus. J Neurophysiol 2005;93:603-608.

44. Schwarzlose RB, Baker Cl, Kanwisher N. Separate face and body selectivity on the fusiform gyrus. J Neurosci 2005;25:11055-11059.

45. Frank GK, Reynolds JR, Shott ME, O’Reilly RC. Altered temporal difference learning in bulimia nervosa. Biol Psychiatry 2011;70:728-735. 